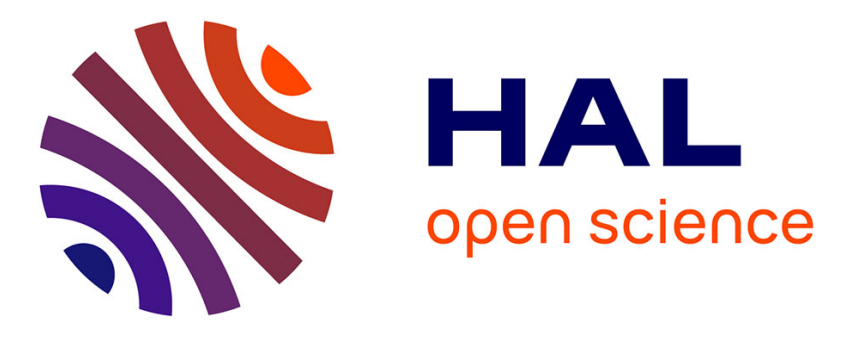

\title{
Transport and dispersion across wiggling nanopores
}

\author{
Sophie Marbach, David S. Dean, Lydéric Bocquet
}

\section{To cite this version:}

Sophie Marbach, David S. Dean, Lydéric Bocquet. Transport and dispersion across wiggling nanopores. Nature Physics, 2018, 14 (11), pp.1108-1113. 10.1038/s41567-018-0239-0 . hal-01990486

\section{HAL Id: hal-01990486 \\ https://hal.science/hal-01990486}

Submitted on 23 Jan 2019

HAL is a multi-disciplinary open access archive for the deposit and dissemination of scientific research documents, whether they are published or not. The documents may come from teaching and research institutions in France or abroad, or from public or private research centers.
L'archive ouverte pluridisciplinaire $\mathbf{H A L}$, est destinée au dépôt et à la diffusion de documents scientifiques de niveau recherche, publiés ou non, émanant des établissements d'enseignement et de recherche français ou étrangers, des laboratoires publics ou privés.

\section{(이)(\$)}

Distributed under a Creative Commons Attribution - NonCommercial - NoDerivatives| 4.0 


\title{
Transport and dispersion across wiggling nanopores
}

\author{
Sophie Marbach', David S. Dean² and Lydéric Bocquet ${ }^{1 *}$
}

\begin{abstract}
The transport of fluids at the nanoscale has achieved major breakthroughs over recent years ${ }^{1-4}$; however, artificial channels still cannot match the efficiency of biological porins in terms of fluxes or selectivity. Pore shape agitation-due to thermal fluctuations or in response to external stimuli-is believed to facilitate transport in biochannels ${ }^{5-9}$, but its impact on transport in artificial pores remains largely unexplored. Here we introduce a general theory for transport through thermally or actively fluctuating channels, which quantifies the impact of pore fluctuations on confined diffusion in terms of the spectral statistics of the channel fluctuations. Our findings demonstrate a complex interplay between transport and surface wiggling: agitation enhances diffusion via the induced fluid flow, but spatial variations in pore geometry can induce a slowing down via entropic trapping, in full agreement with molecular dynamics simulations and existing observations from the literature. Our results elucidate the impact of pore agitation in a broad range of artificial and biological porins, but also, at larger scales, in vascular motion in fungi, intestinal contractions and microfluidic surface waves. These results open up the possibility that transport across membranes can be actively tuned by external stimuli, with potential applications to nanoscale pumping, osmosis and dynamical ultrafiltration.
\end{abstract}

"Everything that living things do can be understood in terms of the jigglings and wigglings of atoms": this quotation by R. P. Feynman highlights the importance of fluctuations in living matter. Transport driven by fluctuations in biological nanopores is no exception to this rule, and has been widely investigated ${ }^{5-9}$. Similar observations have been made in (non-living) fluid phases, where bulk hydrodynamic fluctuations dramatically affect nanoscale dynamics ${ }^{10-12}$. More recently, numerical simulations have noted the impact of phonon modes in carbon nanotubes on the transport of particles confined within them ${ }^{13-15}$. At much larger scales, microfluidic surface waves have been exploited to sort or mix solute particles ${ }^{16}$, and peristalsis in fungal species or in the small intestine significantly modifies nutrient and bacterial transport ${ }^{17,18}$. While these observations-which span a considerable range of length scales-point to a quantitative impact of surface agitation on the transport in confined geometries, a general theory, predicting the dependence of transport properties on the surface fluctuations, is lacking. Even the simple question 'do pore surface fluctuations enhance or diminish transport?' is surprisingly difficult to answer. While surface fluctuations are naively expected to enhance diffusive transport via induced hydrodynamic flows, which underlies the canonical Taylor-Aris mechanism ${ }^{19,20}$, geometrical bulges may entrap particles, resulting in entropic slowing down, as captured by the Fick-Jacobs framework ${ }^{21,22}$, sketched in Fig. 1d. The more general situation of transport with temporally varying geometry remains open despite its obvious importance.

In this paper, we establish a general relationship between diffusive transport and the dynamical spectrum of surface fluctuations. Our framework applies to the case where structural fluctuations of the confining pore are induced by thermal noise, but crucially also to active, non-equilibrium fluctuations induced by external stimuli. We apply our formalism to several situations relevant to nanopore transport ${ }^{13,14}$, as well as to larger-scale configurations, for example active contractions in fungal species, which influence nutrient transport ${ }^{17,23,24}$; see Fig. 1a-c.

Our theory starts with the perturbative analysis of the diffusion of a particle confined between fluctuating surfaces. For simplicity, we consider the two-dimensional geometry presented in Fig. 1e, but the results and the formalism can readily be extended to three dimensions (Supplementary Information 1). The upper surface is located at $z=H+h(x, t)$, where $H$ is the mean height and $h(x, t)$ represents fluctuations about this mean ( $x$ represents the coordinate along the channel and $z$ the height coordinate). The tracer particle-for instance, a molecule of dye in the fluid-locally diffuses with a bare diffusion constant $D_{0}$ and is advected by a hydrodynamic flow $\mathbf{u}$. The probability density $p(x, z, t)$ of the particle obeys the Fokker-Planck (or Smoluchowski) equation: $\partial_{t} p=-\nabla \cdot(\mathbf{u} p)+D_{0} \Delta p$. At this stage, the convective degrees of freedom arising from the membrane fluctuations are not averaged out and explicitly enter the Fokker-Planck equation. The Fokker-Planck equation is valid as long as the timescales associated with the tracer position are larger than the microscopic relaxation timescales, and we can verify that this timescale separation assumption holds in practice down to molecular confinement ${ }^{25}$. In the case of thin channels, a standard expansion (Supplementary Information 1 and 2) yields a reduced advection diffusion equation for the marginal probability density $p^{*}(x, t)=\int_{0}^{H+h(x, t)} p(x, z, t) \mathrm{d} z$,

$$
\partial_{t} p^{*}=-\partial_{x}\left(\bar{u}_{x} p^{*}\right)-D_{0} \partial_{x}\left(\frac{\partial_{x} h}{(H+h)} p^{*}\right)+D_{0} \partial_{x x} p^{*}
$$

where $\bar{u}_{x}=\frac{1}{H+h(x, t)} \int_{0}^{H+h(x, t)} u_{x}(x, z, t) d z$ is the height averaged longitudinal hydrodynamic flow. Incompressibility of the flow also gives $\partial_{x}\left((H+h) \bar{u}_{x}\right)=-\partial_{t} h$. Equation (1) contains two advection terms, which both depend explicitly on height fluctuations. The first is advection by the height-averaged hydrodynamic flow, expected to enhance diffusion. The second term is present even in the absence of any fluid in the channel and represents the effect of geometric fluctuations of the confining channel. When $h$ does not depend on time, the advection term is zero and the second term represents an entropic potential, which slows down diffusion ${ }^{21}$. 


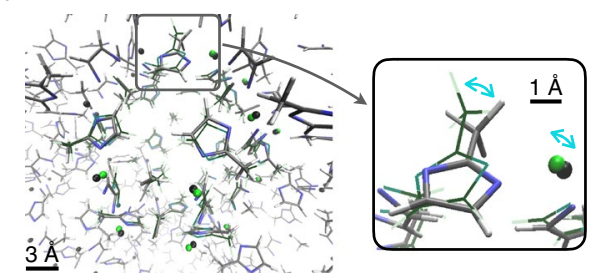

b

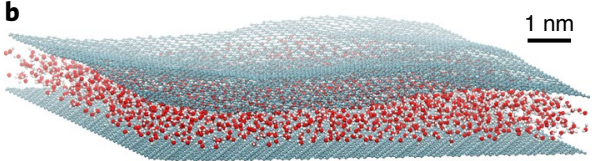

c



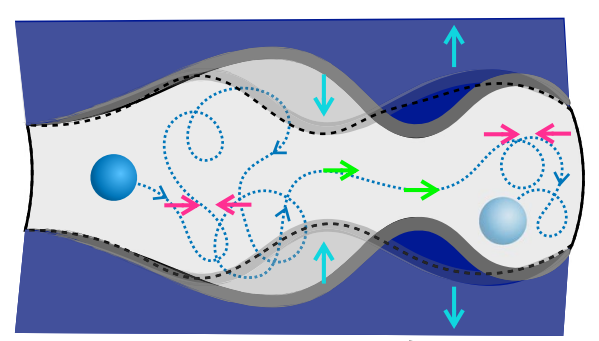

$\rightarrow$ Flow advection

$\rightarrow$ Entropic barrier

Interface motion

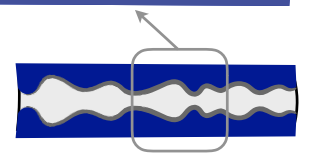

e

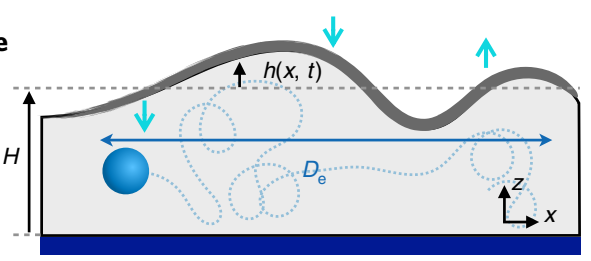

Fig. 1 | Spectral mixing under interface fluctuations. a, Superposition of a unit cell of the zinc imidazolate framework- 8 at $300 \mathrm{~K}$ at two time points separated by 3 ps (grey versus green). The inset shows a magnified view (courtesy of Romain Gaillac) b, Thermal surface ripples on graphene over water. c, Bright-field image of an individual Physarum polycephalum demonstrating active periodic vascular contractions with time, with an amplitude typically ranging from the left image to the right (courtesy of Gabriel Amselem). d, Schematic representation of the main elements of the theoretical model. An interface fluctuates and/or is actively displaced from a reference height. A particle (in light blue) is advected and diffuses within the medium. Transport is modified by advection in the flow induced by the temporal evolution of the interface and the entropic barriers induced by spatial variations of the interface. e, Schematic representation of the reduced two-dimensional geometry studied in the main text.

We analyse equation (1) via a perturbation expansion in the amplitude of the fluctuations of $h$ (with $\langle h\rangle=0$, where $\langle\cdot\rangle$ is the average over the thermal fluctuations). Using a diagrammatic expansion, and to first non-trivial order ${ }^{26}$, we then demonstrate that the renormalized late-time diffusion constant is given in terms of the spectrum of the pore surface fluctuations $S(k, \omega)$, where $k$ and $\omega$ are Fourier frequencies in space and time, respectively, as

$$
D_{\mathrm{e}}=D_{0}\left(1-\frac{1}{H^{2}} \int \frac{\mathrm{d} k \mathrm{~d} \omega}{(2 \pi)^{2}} \frac{\left(D_{0} k^{2}\right)^{2}-3 \omega^{2}}{\left(D_{0} k^{2}\right)^{2}+\omega^{2}} S(k, \omega)\right)
$$

where $S(k, \omega)$ is defined by $\left\langle\tilde{h}(k, \omega) \tilde{h}\left(k^{\prime}, \omega^{\prime}\right)\right\rangle=(2 \pi)^{2} \delta\left(k+k^{\prime}\right)$ $\delta\left(\omega+\omega^{\prime}\right) S(k, \omega)$. Equation (2) is the main result of this work. The full derivation of this key result is reported in Supplementary Information 3. This equation can be generalized to any dimension; it can also be expressed in terms of the response function, related to the spectrum via fluctuation dissipation, here evaluated at an imaginary frequency (Supplementary Information 4). Note also that a similar expression can be obtained for the wave-induced velocity drift (Supplementary Information 3).

At this stage, the formalism is completely general and can be used to compute the renormalized diffusion resulting from any pore or interface motion in terms of the pore or interface fluctuation spectrum. It may be interpreted as a generalization of the Fick-Jacobs formalism to dynamical confinement. It also applies to the case where fluctuations originate from thermal noise, as well as active (non-equilibrium) fluctuations driven by external stimuli.

Equation (2) highlights that the renormalization of the diffusion constant can be either positive or negative depending on the fluctuation spectrum: jiggling can both speed things up and slow things down! Indeed, when the height field is frozen, we have $S(k, \omega)=(2 \pi) \delta(\omega) S_{\mathrm{f}}(k)$, where $S_{\mathrm{f}}(k)$ is the spectral density of the spatial variations of the frozen height. Here we find $D_{\mathrm{e}} / D_{0}=1-\left\langle h^{2}\right\rangle / H^{2}$, in agreement with Fick-Jacobs ${ }^{21,22}$; diffusion is decreased via entropic trapping. When the height fluctuations are very rapid in time, but have a large spatial wavelength, we find $D_{\mathrm{e}} / D_{0}=1+3\left\langle h^{2}\right\rangle / H^{2}$ and diffusion is enhanced via advection coupling. We highlight that these limiting regimes are very general and can be recovered in the case of more complex surface spectra.

In the following we give two example applications of this theory. We first focus on thermal fluctuations of a thin layer. Thin-film dynamics can be analysed within the lubrification approximation ${ }^{27}$ and in the limit of low Reynolds numbers. The fluctuation interface spectrum can be obtained from the Hamiltonian $\mathcal{H}[h]$ of the height fluctuations $h$. The energy functional depends on the Van der Waals interactions between the surfaces, possibly the discrete properties of the confined liquid ${ }^{28,29}$ and also contributions associated with constraints fixing the average gap size. The dynamics for small height fluctuations are given by ${ }^{27}$

$$
\partial_{t} h=-\partial_{x}\left((H+h) \bar{u}_{x}\right)=\partial_{x}\left(\lambda(H) \partial_{x} \frac{\delta \mathcal{H}}{\delta h}+\sqrt{\lambda(H)} \mathcal{N}\right)
$$

with $\lambda(H)$ the permeance of the slit. The term $\mathcal{N}$ is a Gaussian white noise with $\left\langle\mathcal{N}(x, t) \mathcal{N}\left(x^{\prime}, t^{\prime}\right)\right\rangle=2\left(k_{\mathrm{B}} T / W\right) \delta\left(x-x^{\prime}\right) \delta\left(t-t^{\prime}\right)$, where $T$ is the temperature and $W$ the channel width, generated by hydrodynamic fluctuations within the liquid ${ }^{27}$. Focusing more specifically on a thin film confined by an elastic membrane, the Hamiltonian takes the Landau form

$$
H[h]=\phi(H) W L+\frac{W}{2} \int_{0}^{L} \mathrm{~d} x\left(\phi^{\prime \prime}(H) h^{2}+\kappa(H)\left(\partial_{x x} h\right)^{2}\right)
$$

where $\phi(H)$ is the free energy per unit area of a flat interface (verifying $\phi^{\prime}(H)=0$ for the equilibrium height) and $\kappa(H)$ can be interpreted as the surface's bending rigidity; $L$ is the channel length. In this case $S(k, \omega)=2 k_{\mathrm{B}} T \lambda(H) k^{2} /\left(\omega^{2}+k^{4} \lambda(H)^{2}\left(\phi^{\prime \prime}+\kappa k^{4}\right)^{2}\right)$ and, directly, one finds using equation (2) 

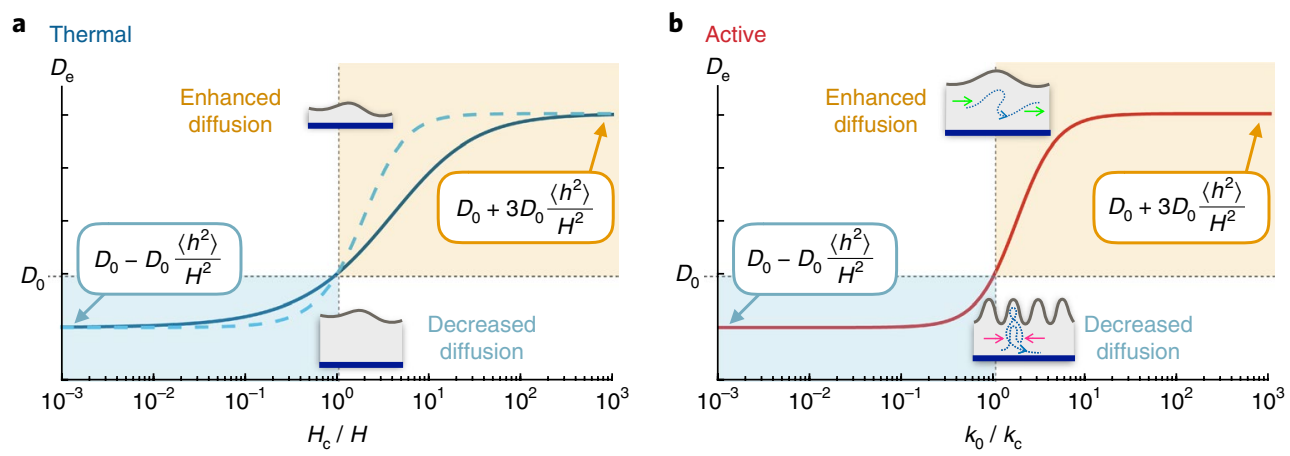

Fig. 2 | Renormalized diffusion under thermally fluctuating and actively driven surfaces. a, Renormalized diffusion $D_{\mathrm{e}}$ for a thermally fluctuating interface, as a function of the average upper interface height $H$. The vertical axis tick marks represent a change of one unit of $D_{0}\left\langle h^{2}\right\rangle H^{2}$. The solid line is for no-slip boundary conditions and the dashed one is for a large slip length on both sides. $\phi$ is assumed, for simplicity, to be accounted for by a Hamaker expression, $\phi(H)=P H+A / 12 \pi H^{2}$ with $A$ a Hamaker constant and $P$ a constant external pressure that fixes the average film height. $H_{c}=\frac{15 A}{24 \pi \eta D_{0}}$ is the critical height such that below (above) this height diffusion is enhanced (decreased). For a water film typically $A=2 \times 10^{-20} \mathrm{~J}^{43}$, and $H_{\mathrm{c}}=4 \mathrm{~nm}$. For slipping surfaces $H_{c}^{2} \simeq \frac{15 A}{4 \pi \eta D_{0}} b$ for $b \gg H$, with a slip length $b \sim 10 \mathrm{~nm}^{25}, H_{c} \simeq 15 \mathrm{~nm}$. b. Renormalized diffusion $D_{\mathrm{e}}$ under an actively fluctuating interface: $D_{\mathrm{e}}$ is plotted as a function of the excitation wavenumber $k_{0} . k_{c}$ is the critical wavenumber defined as $k_{c}^{2}=\omega_{0} / \sqrt{3} D_{0}$. For capillary waves on shallow water, one has $\omega_{0}^{2}=\gamma k_{0}^{3} / \rho_{0^{\prime}}$ where $\rho_{0}$ is the density of water, such that $k_{c}=3 \gamma / \rho D_{0}^{2}$. Typically, $k_{c} \sim 10^{13} \mathrm{~m}^{-1}$ and we expect that capillary waves always enhance mixing. In many other cases the dispersion relation may be more complex, yielding a rich zoology of behaviours.

$$
D_{\mathrm{e}}=D_{0}\left(1+\frac{\left\langle h^{2}\right\rangle}{H^{2}}\left[\frac{4}{\left(1+D_{0} / D(H)\right)^{3 / 4}}-1\right]\right)
$$

with $\left\langle h^{2}\right\rangle=k_{\mathrm{B}} T \ell^{3} / 2 \sqrt{2} W \kappa$, where $\ell=\left(\kappa / \phi^{\prime \prime}(H)\right)^{1 / 4}$ is the characteristic wavelength of the surface fluctuations. The quantity $D(H)=\lambda(H) \phi^{\prime \prime}(H)$ is a diffusion coefficient scale associated with interface fluctuations. For no slip boundary, one has $D(H)=\frac{H^{3}}{12 \eta} \phi^{\prime \prime}(H)$, where $\eta$ is the viscosity of the liquid. Other fluctuating confinement cases, with different Hamiltonians, geometries (e.g. tubes) and boundary conditions (e.g. partial slip), can easily be analysed, and further examples are given in Supplementary Information 6, leading to qualitatively similar conclusions.

Equation (5) shows that if bulk diffusion is large (specifically, $D_{0} / D(H) \geq \mathcal{M}_{0}$ with $\mathcal{M}_{0}=4^{4 / 3}-1$ ), then effective diffusion will be reduced, i.e. $D_{\mathrm{e}} \leq D_{0}$. Particles with high bare diffusivity see an effectively quenched surface profile, which generates an entropic trapping potential, thus slowing down diffusion. On the other hand, for slowly diffusing particles, $D_{0} / D(H) \leq \mathcal{M}_{0}$, the fluctuationinduced advection enhances diffusion, i.e. $D_{\mathrm{e}} \geq D_{0}$. This results in a compensation effect, summarized in Fig. 2a, whereby fast particles are slowed down and slow particles are sped up. This difference in effective diffusion as a function of bare diffusion has been observed in simulations of gas particles in metal-organic frameworks ${ }^{30}$ and water transport in biological porins ${ }^{9}$. Equation (5) also describes typical low-inertia phonon modes on an interface (such as phonons on the surface of graphene or carbon nanotubes ${ }^{31}$; see also Supplementary Information 7). The impact of phonon modes of a carbon nanotube on confined water has indeed been measured and shown to depend substantially on phonon frequency ${ }^{13}$.

As a second example, we consider a situation where the fluctuations of the pore's shape are driven out of equilibrium by an external stimulus. This situation is particularly interesting in the sense that the pore structure undergoes non-equilibrium fluctuations, therefore leading to out-of-equilibrium transport. In general, this situation can be accounted for by adding a force to equation (3) with a specific frequency $\omega_{0}$ and wavenumber $k_{0}$. We consider here a propagating wave of amplitude $a_{0}$ on the interface of a deforming tube with bare radius $R$ as $\delta R(x, t)=a_{0} \cos \left(\omega_{0} t-k_{0} x\right)$. In this context, the relevant driving spectrum is given by $S(k, \omega)=\pi^{2} a_{0}^{2}\left[\delta\left(\omega_{0}+\omega\right) \delta\right.$ $\left(k_{0}-k\right)+\delta\left(\omega_{0}-\omega\right) \delta\left(k_{0}+k\right)$ ], and using the result of equation (2) for a tubular geometry we find (see Supplementary Information 5 and 8 for the exhaustive derivation)

$$
D_{\mathrm{e}}=D_{0}\left(1-\frac{a_{0}^{2}}{2 R^{2}} \frac{1-3\left(D_{\approx} / D_{0}\right)^{2}}{1+\left(D_{\approx} / D_{0}\right)^{2}}\right)
$$

where $D_{\approx}=\omega_{0} / k_{0}^{2}$ can be interpreted as a diffusion coefficient associated with the surface wavepacket. Interestingly, one recovers, from equation (6), the two regimes highlighted previously, but now for the actively fluctuating surfaces: diffusion is enhanced or slowed down depending on whether the bare diffusion $D_{0}$ is lower or higher than the wavepacket diffusion $D_{\approx}$. The results are summarized in Fig. 2b.

Altogether, for the in- and out-of-equilibrium cases respectively, one can define a dimensionless Péclet-like number to characterize the transition from decreased to enhanced mixing. For equilibrium fluctuations $P e=D(H) / D_{0}$ with $D(H)$ the dispersion induced by surface-induced flow, while for active fluctuations $P e=D_{\approx} / D_{0}$, introducing active surface transport via $D_{\approx}$. One may accordingly expect that $P e \sim \ell^{2} / D_{0} \tau$ where $\ell$ and $\tau$ are characteristic length and time scales of the fluctuations. An outcome of our theoretical framework is that this Péclet number enables us to quantify the impact of surface fluctuations on the transport. Typically, for high (low) Péclet number $P e \geq 1(P e \leq 1)$, the transport is expected to increase (decrease) under structural jiggling; see Fig. 3.

We show in Fig. 3 a variety of systems where the structural agitation of the confinement is expected to have an impact on particle transport. These fluctuating systems are classified according to their corresponding Péclet number (full details are given in Supplementary Information 10). (1) Biological channels, such as aquaporins, will undergo thermal fluctuations with typically $P e \sim 10^{-2}-10^{2}$ (Supplementary Table 1), indeed both behaviours were observed for water in a KcsA channel ${ }^{9}$, and diffusion is indeed enhanced where fluctuation timescales are fast. In the selectivity filter of the KcsA channel, fluctuations are typically $0.5-0.8 \AA$, so the enhancement factor is $a_{0}^{2} / R^{2} \simeq 0.1-0.2^{6,32}$. (2) In metal-organic frameworks the range is similar (Supplementary Table 2): small gas molecules such as $\mathrm{H}_{2}$ have $P e \leq 1$, and indeed such molecules are not accelerated by framework flexibility ${ }^{30}$; while large gas molecules have $P e \geq 1$, and indeed molecular diffusion is increased, well 


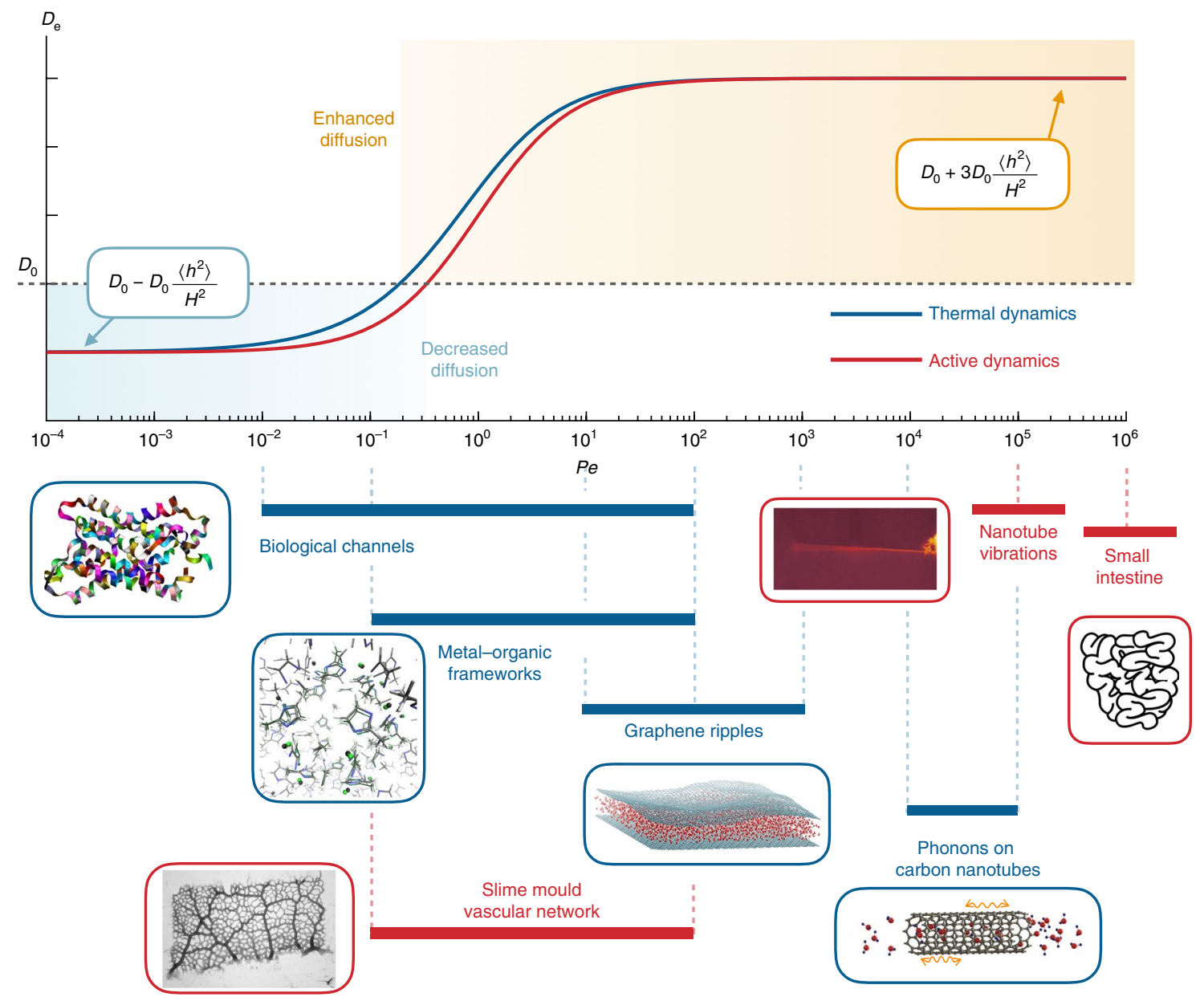

Fig. 3 | Enhanced or decreased transport under pore shape wiggling versus the dimensionless Péclet-like number for various fluid transporters. Effective diffusion, in thermally or actively fluctuating pores, renormalized by thermal dynamics spectrum described by equation (5) or by active dynamics described by equation (6). The diagram compares different fluid transporters, as described in the main text. (Aquaporin for biological channels thanks to ref. ${ }^{44}$, slime mould courtesy of Karen Alim and vibrating nanotube from ref. ${ }^{45}$ ).

beyond perturbation theory, e.g. $D_{\mathrm{e}} / D_{0} \gg 1$. In this context, the spatial and temporal vibrations yield $\left\langle h^{2}\right\rangle / H^{2} \simeq 0.3^{33}$ and thus a significant enhancement of $D_{\mathrm{e}}$. (3) Graphene sheets show thermal ripples that are expected to affect water transport, typically with $\mathrm{Pe} \sim 10^{1}-10^{3}$ (Supplementary Table 3 ). We have fully confirmed the theoretical picture in this situation by carrying out our own molecular dynamics simulations of water confined between graphene sheets: the thermally fluctuating sheets do increase particle diffusion by $150 \%$ as compared to the flat rigid sheets, while diffusion between undulated but frozen graphene sheets is reduced to $80 \%$ with respect to the flat rigid sheets; see Supplementary Information 11. Putting in the numbers for the fluctuating graphene case, the typical enhancement factor for $D_{\mathrm{e}}$ in our simulations is actually found to be larger than the prediction $1+3\left\langle h^{2}\right\rangle / H^{2} \simeq 110 \%$, pointing to complementary molecular effects. (4) Finally, we find that non-dissipative phonon coupling is in the range $\mathrm{Pe} \sim 10^{4}-10^{5}$ (Supplementary Table 4) and always enhances diffusion, in accordance with the observations of ref. ${ }^{13}$.

Moving to actively driven dynamics, various examples include (1) nanotubes, in which longitudinal mechanical vibrations could typically yield $P e \sim 10^{5}$ (Supplementary Table 5), echoing the numerical results of ref. ${ }^{34}$, (2) the small intestine, which demonstrates contractile activity with typically $P e \sim 10^{6}$ (Supplementary Table 6) (evidence of an increased dispersion has also been observed, though not quantified, in a microfluidic device mimicking the contractions of the intestine ${ }^{18}$; in the small bowel, one finds $a_{0}^{2} / R^{2} \simeq 0.12-0.25$ and thus the enhancement of $D_{\mathrm{e}}$ is expected to be large $\mathrm{e}^{35}$ ), and (3) slime mould vascular networks, in which nutrient dispersion is characterized typically by $P e \sim 10^{-1}-10^{2}$, and increasing for larger organisms (Supplementary Table 7). The latter example of nutrient dispersal in contracting vascular networks of organisms is actually of particular interest, since it is essential for survival to generate alternative strategies for fast nutrient dispersal. For example the unicellular organism $P$. polycephalum, shown in Fig. 1c, is composed of a connected network of veins, containing cytoplasm that can deliver nutrients to the entire body: it exhibits non-stationary periodic shuttle flows ${ }^{36}$ driven by a peristaltic wave of contractions ${ }^{17}$ spanning the organism. For an individual such as the one in Fig. 3 with length $L=8 \mathrm{~mm}, k_{0} \simeq 2 \pi / L \sim 800 \mathrm{~m}^{-1}$ and $\omega_{0} \sim 0.06 \mathrm{~s}^{-136}$, we find $D_{\approx} \sim 10^{-7} \mathrm{~m}^{2} \mathrm{~s}^{-1}$. This is two orders of magnitude larger than the bare diffusion of nutrient molecules, with at most $D_{0} \sim 10^{-9} \mathrm{~m}^{2} \mathrm{~s}^{-1}$, as indeed observed in simulations in ref. ${ }^{17}$. In this organism the hierarchical structure of veins leads to $a_{0} / R \gtrsim 0.9$ ( $a_{0}$ being the typical difference between large and small veins), such that the enhancement of $D_{\mathrm{e}}$ is expected to be large.

This figure highlights the fact that a wide variety of situations, covering a huge range of scales, can be put in perspective under the framework of spectral mixing by confinement jiggling. Going further, it is possible to study the relative effect of coupled passive and active dynamics, for instance in biological membranes, with an 
active component ${ }^{37}$, or nanoscale systems such as nanotubes that are sensitive to thermal as well as activated vibrations ${ }^{38}$. Taking a step further, it would be interesting to extend the theory and investigate how coloured noise or nanoscale non-Markovian effects might influence transport. Moving to macroscopic scales, it would be interesting to connect this formalism with cases where the flow velocity presents some recirculation, as may occur in the intestine ${ }^{18}$ but also in ocean waves, which have been shown to influence mixing and dispersion ${ }^{39}$.

Our formalism allows us, in particular, to identify the key components to design active channels. The results here show that it is possible to actively tune the diffusion of species, therefore dynamically differentiating solutes as a function of their own Peclet number, and thus providing the conditions for active separation ${ }^{40}$. Moreover, the modification of osmosis processes in fluctuating channels remains to be explored, along with their applications to filtration and desalination. In a different context, to improve the sensitivity of sequencing via translocation through nanopores, a slowing down of some species may indeed be required. Our results also demonstrate that this fine tuning is possible by harnessing out-of-equilibrium dynamics of the pore, with considerable implications for DNA sequencing ${ }^{41,42}$.

Data availability. The data that support the plots within this paper and other findings of this study are available from the corresponding author upon reasonable request.

\section{Acknowledgements}

The authors are indebted to B. Rotenberg for several fruitful discussions on molecular dynamics, and to K. Alim for bringing to the discussion biologically related examples. The authors also thank F.-X. Courdert, D.avid Lacoste and J.-F. Joanny for interesting discussions. S.M. acknowledges funding from a J.-P. Aguilar grant of the CFM foundation. D.S.D. acknowledges funding from the ANR grant FISICS. L.B. acknowledges support from ANR grant Neptune. This work was granted access to the HPC resources of MesoPSL financed by the Region Ile de France and the project Equip@ Meso (reference ANR-10-EQPX-29-01) of the programme Investissements d'Avenir supervised by the Agence Nationale pour la Recherche.

\section{Author contributions}

L.B. designed the research. S.M., D.S.D. and L.B. conducted research. S M. carried out the molecular dynamics simulations. S M., D.S.D. and L.B. wrote the paper.

\section{Competing interests}

The authors declare no competing interests.

\section{References}

1. Feng, J. et al. Single-layer $\mathrm{MoS}_{2}$ nanopores as nanopower generators. Nature 536, 197-200 (2016).

2. Secchi, E. et al. Massive radius-dependent flow slippage in carbon nanotubes. Nature 537, 210-213 (2016).

3. Tunuguntla, R. H. et al. Enhanced water permeability and tunable ion selectivity in subnanometer carbon nanotube porins. Science 357, 792-796 (2017).

4. Esfandiar, A. et al. Size effect in ion transport through angstrom-scale slits. Science 358, 511-513 (2017).

5. Wand, A. J. Dynamic activation of protein function: a view emerging from NMR spectroscopy. Nat. Struct. Mol. Biol. 8, 926-931 (2001).

6. Noskov, S. Y., Berneche, S. \& Roux, B. Control of ion selectivity in potassium channels by electrostatic and dynamic properties of carbonyl ligands. Nature 431, 830-834 (2004)

7. Bhabha, G. et al. A dynamic knockout reveals that conformational fluctuations influence the chemical step of enzyme catalysis. Science 332, 234-238 (2011).

8. Wei, G., Xi, W., Nussinov, R. \& Ma, B. Protein ensembles: how does nature harness thermodynamic fluctuations for life? The diverse functional roles of conformational ensembles in the cell. Chem. Rev. 116, 6516-6551 (2016).

9. Allen, T. W., Kuyucak, S. \& Chung, S.-H. Molecular dynamics study of the KcsA potassium channel. Biophys. J. 77, 2502-2516 (1999).

10. Moseler, M. \& Landman, U. Formation, stability, and breakup of nanojets. Science 289, 1165-1169 (2000).

11. Davidovitch, B., Moro, E. \& Stone, H. A. Spreading of viscous fluid drops on a solid substrate assisted by thermal fluctuations. Phys. Rev. Lett. 95, 244505 (2005).
12. Fetzer, R., Rauscher, M., Seemann, R., Jacobs, K. \& Mecke, K. Thermal noise influences fluid flow in thin films during spinodal dewetting. Phys. Rev. Lett. 99, 114503 (2007).

13. Ma, M. et al. Water transport inside carbon nanotubes mediated by phonon-induced oscillating friction. Nat. Nanotech 10, 692-695 (2015).

14. Ma, M., Tocci, G., Michaelides, A. \& Aeppli, G. Fast diffusion of water nanodroplets on graphene. Nat. Mater. 15, 66 (2016).

15. Cruz-Chú, E. R. et al. On phonons and water flow enhancement in carbon nanotubes. Nat. Nanotech. 12, 1106 (2017).

16. Yeo, L. Y. \& Friend, J. R. Surface acoustic wave microfluidics. Annu. Rev. Fluid Mech. 46, 379-406 (2014).

17. Alim, K., Amselem, G., Peaudecerf, F., Brenner, M. P., \& Pringle, A. Random network peristalsis in Physarum polycephalum organizes fluid flows across an individual. Proc. Natl Acad. Sci. USA 110, 13306-13311 (2013).

18. Cremer, J. et al. Effect of flow and peristaltic mixing on bacterial growth in a gut-like channel. Proc. Natl Acad. Sci. USA 113, 11414-11419 (2016).

19. Taylor, G. Dispersion of soluble matter in solvent flowing slowly through a tube. Proc. R. Soc. London Ser. A 219, 186-203 (1953).

20. Aris, R. On the dispersion of a solute in a fluid flowing through a tube. Proc. R. Soc. Lond. A 235, 67-77 (1956).

21. Reguera, D. \& Rubi, J. Kinetic equations for diffusion in the presence of entropic barriers. Phys. Rev. E 64, 061106 (2001).

22. Malgaretti, P., Pagonabarraga, I. \& Rubi, J. M. Entropic electrokinetics: recirculation, particle separation, and negative mobility. Phys. Rev. Lett. 113, 128301 (2014).

23. Kuroda, S., Takagi, S., Nakagaki, T. \& Ueda, T. Allometry in Physarum plasmodium during free locomotion: size versus shape, speed and rhythm. J. Exp. Biol. 218, 3729-3738 (2015).

24. Tlalka, M., Bebber, D., Darrah, P., Watkinson, S. \& Fricker, M. Emergence of self-organised oscillatory domains in fungal mycelia. Fungal Genet. Biol. 44, 1085-1095 (2007).

25. Bocquet, L. \& Charlaix, E. Nanofluidics, from bulk to interfaces. Chem. Soc. Rev. 39, 1073-1095 (2010).

26. Démery, V. \& Dean, D. S. Perturbative path-integral study of active- and passive-tracer diffusion in fluctuating fields. Phys. Rev. E 84, 011148 (2011).

27. Grün, G., Mecke, K. \& Rauscher, M. Thin-film flow influenced by thermal noise. J. Stat. Phys. 122, 1261-1291 (2006).

28. Stubenrauch, C. \& Von Klitzing, R. Disjoining pressure in thin liquid foam and emulsion films? New concepts and perspectives. J. Phys. Condens. Matter 15, R1197 (2003).

29. Gravelle, S., Ybert, C., Bocquet, L. \& Joly, L. Anomalous capillary filling and wettability reversal in nanochannels. Phys. Rev. E 93, 033123 (2016).

30. Haldoupis, E., Watanabe, T., Nair, S. \& Sholl, D. S. Quantifying large effects of framework flexibility on diffusion in MOFs: $\mathrm{CH}_{4}$ and $\mathrm{CO}_{2}$ in ZIF-8. ChemPhysChem 13, 3449-3452 (2012).

31. Thomas, J. A., Turney, J. E., Iutzi, R. M., Amon, C. H. \& McGaughey, A. J. Predicting phonon dispersion relations and lifetimes from the spectral energy density. Phys. Rev. B 81, 081411 (2010).

32. Compoint, M., Carloni, P., Ramseyer, C. \& Girardet, C. Molecular dynamics study of the KcsA channel at 2.0-A resolution: stability and concerted motions within the pore. Biochim. Biophys. Acta Biomembr. 1661, 26-39 (2004).

33. Park, K. S. et al. Exceptional chemical and thermal stability of zeolitic imidazolate frameworks. Proc. Natl Acad. Sci. USA 103, 10186-10191 (2006).

34. Qiu, H., Shen, R. \& Guo, W. Vibrating carbon nanotubes as water pumps. Nano Res. 4, 284-289 (2011)

35. Froehlich, J. M. et al. Small bowel motility assessment with magnetic resonance imaging. J. Magn. Reson. Imaging 21, 370-375 (2005).

36. Stewart, P. A. \& Stewart, B. T. Protoplasmic movement in slime mold plasmodia: the diffusion drag force hypothesis. Exp. Cell Res. 17, 44 (1959).

37. Girard, P., Prost, J. \& Bassereau, P. Passive or active fluctuations in membranes containing proteins. Phys. Rev. Lett. 94, 088102 (2005).

38. Sazonova, V. et al. A tunable carbon nanotube electromechanical oscillator. Nature 431, 284-287 (2004).

39. Herterich, K. \& Hasselmann, K. The horizontal diffusion of tracers by surface waves. J. Phys. Oceanogr. 12, 704-711 (1982).

40. Marbach, S. \& Bocquet, L. Active sieving across driven nanopores for tunable selectivity. J. Chem. Phys. 147, 154701 (2017).

41. Kowalczyk, S. W., Wells, D. B., Aksimentiev, A. \& Dekker, C. Slowing down DNA translocation through a nanopore in lithium chloride. Nano. Lett. 12, 1038-1044 (2012).

42. Keyser, U. F. Controlling molecular transport through nanopores. J. R. Soc. Interface 12, 1369-1378 (2011).

43. Israelachvili, J. N. \& Adams, G. E. Measurement of forces between two mica surfaces in aqueous electrolyte solutions in the range $0-100 \mathrm{~nm}$. J. Chem. Soc. Faraday Trans. 1 74, 975-1001 (1978).

44. Berman, H. M. et al. in International Tables for Crystallography Vol. F (eds Rossman, M. G. \& Arnold, E.) 675-684 (Springer, Dordrecht, 2006).

45. Siria, A. \& Niguès, A. Electron beam detection of a nanotube scanning force microscope. Sci. Rep. 7, 11595 (2017). 